\title{
Molecular weight and tacticity effect on morphological and mechanical properties of Ziegler-Natta catalyzed isotactic polypropylenes
}

\author{
Ismael Amer ${ }^{1,2 *}$, Albert van Reenen ${ }^{1}$ and Touhami Mokrani ${ }^{2}$ \\ ${ }^{1}$ Department of Chemistry and Polymer Science, University of Stellenbosch, Stellenbosch, South Africa \\ ${ }^{2}$ Department of Civil and Chemical Engineering, College of Science, Engineering and Technology, \\ University of South Africa - UNISA, Johannesburg, South Africa \\ *ismaelamer77@yahoo.co.za
}

\begin{abstract}
The morphological and mechanical properties of Ziegler-Natta catalyzed isotactic polypropylenes as influenced by the molecular weight and tacticity was investigated. Polypropylene samples were injection moulded into standard disks with a HAAKE MiniJet II injection moulder at $190{ }^{\circ} \mathrm{C}$ and 200 bar for morphological and mechanical tests. The morphological and mechanical properties of specimens were investigated by means of optical microscope (OM), scanning electron microscopy (SEM), microhardness (MH) and dynamic mechanical analysis (DMA). The samples exhibited a typical $\alpha$-modification spherulite structure of isotactic polypropylenes crystallized from the melt. It was found that the most important factor affecting the structure and properties of these polymers is the isotacticity content. A clear molecular weight effect was also found for samples with low molecular weights. The microhardness and storage modulus values increased as crystallinity did. Accordingly, isotacticity degree is considered as the main parameter affecting the crystallinity of samples.
\end{abstract}

Keywords: mechanical property, molecular weight, morphological property, polypropylene, tacticity.

\section{Introduction}

Polypropylene is one of the most common polymers in use today. Its good mechanical properties and relatively low price result in the continuous growth of its production and the expansion of its market. Its continuously increasing application accelerates research in all related fields, including the preparation of isotactic polypropylene based composites and blends ${ }^{[1]}$. The mechanical and physical properties of polypropylene are influenced by a number of factors. The mechanical properties of the majority of polypropylene homopolymers are, apart from processing conditions, influenced by their rheological and crystallization behaviour. The degree of crystallinity is considered to be the most influential property affecting the physical and mechanical properties of a polypropylene sample ${ }^{[2-4]}$. An increase in crystallinity is often related to an increase in properties such as the stiffness or storage modulus of a sample, while other factors such as the impact strength generally decrease with increasing crystallinity. The storage modulus can be defined as the ratio of stress to strain under vibratory conditions which can be calculated from data obtained from either free or forced vibration tests, in shear, compression, or elongation. The Stiffness can be defined as the rigidity of the material to which it resists deformation in response to an applied force. An increase in crystallinity can lead to an increase in the lamellar thickness which leads to higher storage modulus and stiffiness values ${ }^{[2-4]}$. The effects of molecular weight $^{[5-8]}$, molecular weight distribution ${ }^{[9]}$ and tacticity ${ }^{[10-13]}$ on the crystallization have been investigated by several authors. Cheng et al. ${ }^{[6]}$ showed that the linear growth rate of crystals decreases with the increase of molecular weight, but the overall crystallization rate might increase because an increasing number of intramolecular folded-chain nuclei could result in a higher nucleation density ${ }^{[7]}$. For samples with similar molecular weights and different tacticities the linear crystal growth rate might increase by three orders of magnitude when the isotacticity ( $\mathrm{mmmm} \%$ ) of isotactic polypropylene increases from 78.7 to $98.8 \%^{[11,14]}$. The degree of crystallinity of isotactic polypropylene is commonly in the range of 40 to $70 \%{ }^{[1]}$. Atactic polypropylene, on the other hand, is considered uncrystallizable, since the chain structure lacks regularity. Isotactic polypropylene can crystallize in three different crystal forms as was described by Bruckner et al. ${ }^{[15]}$, depending on the polymer structure and the crystallization conditions: the $\alpha$-form with a monoclinic, the $\gamma$-form with an orthorhombic and the $\beta$-form with a hexagonal unit cell ${ }^{[15]}$.

The molecular weight has also been shown to influence the glass transition temperature $\left(\mathrm{T}_{\mathrm{g}}\right)$ of polymers, with higher molecular weight samples having a higher $\mathrm{T}_{\mathrm{g}}{ }^{[16]}$. This in turn influences the mobility of chains at room temperature, and since polypropylene has a $\mathrm{T}_{\mathrm{g}}$ range in the region of $0{ }^{\circ} \mathrm{C}$, variations in the $\mathrm{T}_{\mathrm{g}}$ temperature range can have an effect on the ability of the material to displace energy at low temperatures.

The aim of the current study is to explore narrowly the structure-property relationships of polypropylene. The morphological and mechanical properties of different samples were investigated by means of optical microscope (OM), scanning electron microscopy (SEM), microhardness 
(MH) and dynamic mechanical analysis (DMA) to demonstrate the effect of isotacticity and molecular weight on the properties of polypropylene samples. To the best of our knowledge the investigation of how the molecular weight affect the thermal, morphological and mechanical properties of isotactic polypropylene has not been done yet to polymer studies which has previously been overlooked.

\section{Experimental Section}

\subsection{Materials}

Polypropylene polymers and fractions used in this study were selected from our previous study as shown in Amer and van Reenen ${ }^{[17]}$ and Table 1 . These polymers were prepared using a commercial Ziegler-Natta catalyst with $2.78 \mathrm{wt} \%$ Ti content purchased from Star Chemicals \& Catalysts Co. (China) and labeled as P3-P17. P4 ${ }^{(120)}, \mathrm{P}^{(120)}, \mathrm{P} 8^{(120)}$ and $\mathrm{P} 9^{(110)}$ refer to polypropylene TREF fractions eluted at 120 and $110^{\circ} \mathrm{C}$, respectively.

\subsection{Polymerization procedure}

All polymerization reactions were carried out under an inert gas atmosphere. The polymerization reactions were carried out in a $350-\mathrm{mL}$ stainless steel Parr autoclave with a gas inlet and pressure gauge. Typically the reactor was charged with the catalyst ( $43 \mathrm{mg}$, Ti content $2.78 \mathrm{wt} \%$ ) and triethylaluminium ( $2 \mathrm{mmol}, \mathrm{Al} / \mathrm{Ti}$ mole ratio 80 ) in toluene $(25 \mathrm{~mL})$. The catalyst solution was stirred for $5 \mathrm{~min}$ and then the propylene was added. The reactor was pressurized with hydrogen and the contents stirred for $1 \mathrm{~h}$ at room temperature. The reaction was then quenched by the addition of $100 \mathrm{ml}$ $10 \% \mathrm{HCl} / \mathrm{MeOH}$. The resulting polymer was filtered off, washed several times with methanol, and subsequently dried under vacuum at $80^{\circ} \mathrm{C}$ for $15 \mathrm{~h}$, to yield about $3-5 \mathrm{~g}$ of polypropylene as a white powder.

\subsection{Sample preparation for mechanical tests}

Test specimens were injection moulded into standard disks for morphological and mechanical tests with a HAAKE MiniJet II injection moulder. The injection moulding temperature was $190{ }^{\circ} \mathrm{C}$ and the injection pressure was $200 \mathrm{bar}$. The dimensions of the standard disks are $20.0 \mathrm{~mm}$ in diameter and $1.5 \mathrm{~mm}$ in thickness.

\subsection{Preparation of etching reagent}

Permanganic etching of polyolefins was used to prepare samples for the study of the morphology. This technique has been used in several studies involving polyolefin ${ }^{[18-21]}$. Potassium permanganate (1 g) (obtained from Sigma-Aldrich) was dissolved in $100 \mathrm{~mL}$ of a concentrated solution of 33 vol \% phosphoric acid and $67 \mathrm{vol} \%$ sulphuric acid (Sigma-Aldrich). The solution was prepared by adding potassium permanganate very slowly to the beaker containing both acids, with rapid agitation. After adding all the potassium permanganate, the beaker was closed and the content stirred until all the potassium permanganate was dissolved (a dark green purple solution formed). All polypropylene samples were etched at room temperature.

\subsection{Etching procedure}

Specimens from each polymer, with approximate dimensions of length $10 \mathrm{~mm}$, width $5 \mathrm{~mm}$ and thickness $1.5 \mathrm{~mm}$, were cut from the disks prepared by injection moulding. Each sample was immersed in about $10 \mathrm{~mL}$ of the etching reagent in a beaker for a period of 60 minutes. This permanganic acid solution preferentially etches the amorphous part of the polymer in the spherulites in such a way that the lamellae then appear clearly. Subsequently, the specimens were carefully washed with hydrogen peroxide, distilled water and acetone, in order to avoid any artefacts caused by pollution effects. Samples were finally dried in a vacuum oven at $45^{\circ} \mathrm{C}$ for $5 \mathrm{hr}$.

\subsection{Polymer characterization}

A Zeiss Axiolab OM, (magnification $\times 50-100 \mu \mathrm{m})$ with a high resolution camera CCD-IRIS (Sony) was used to examine the etched piece, to investigate the crystal structure.

SEM analysis of etched piece was performed using a

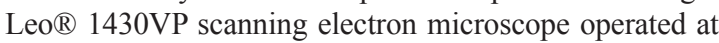
$15 \mathrm{kV}$ of acceleration voltage at room temperature. All the surfaces to be studied were coated with gold under vacuum in order to eliminate any undesirable charge effects during the SEM observations.

Samples for compressive DMA were analyzed using a Perkin Elmer DMA 7e calibrated according to standard procedures. The samples were first melted at $180^{\circ} \mathrm{C}$ for 8 minutes and then melt pressed at $5 \mathrm{MPa}$ and same temperature. The samples were analyzed using a $5{ }^{\circ} \mathrm{C} / \mathrm{min}$

Table 1. Characterization data of the polypropylenes and fractions.

\begin{tabular}{|c|c|c|c|c|c|c|c|}
\hline Samples & $M_{w}{ }^{a}(g / m o l)$ & $\mathbf{M}_{\mathbf{w}} / \mathbf{M}_{\mathrm{n}}$ & $m m m m^{\mathrm{b}}(\%)$ & $\mathbf{T}_{\mathrm{m}}{ }^{\mathrm{c}}\left({ }^{\circ} \mathrm{C}\right)$ & $\mathrm{T}_{\mathrm{c}}^{\mathrm{c}}\left({ }^{\circ} \mathrm{C}\right)$ & $\Delta H_{m}{ }^{c}(J / g)$ & $X_{c}(\%)$ \\
\hline P3 & 184759 & 6.1 & 93.0 & 161.2 & 124.2 & 104.5 & 50.0 \\
\hline $\mathrm{P} 4$ & 252956 & 5.4 & 94.0 & 160.6 & 116.5 & 103.9 & 50.0 \\
\hline P5 & 312580 & 4.1 & 96.0 & 161.9 & 118.4 & 108.9 & 52.0 \\
\hline P8 & 228960 & 6.4 & 94.0 & 162.0 & 124.4 & 103.9 & 50.0 \\
\hline $\mathrm{P} 14$ & 215397 & 5.9 & 86.0 & 157.5 & 119.8 & 90.5 & 43.0 \\
\hline P17 & 65498 & 8.2 & 93.0 & 156.5 & 120.8 & 100.5 & 48.0 \\
\hline $\mathrm{P} 4^{(120)}$ & 195693 & 4.3 & 98.0 & 160.0 & 116.0 & 119.7 & 57.0 \\
\hline $\mathrm{P} 5^{(120)}$ & 207823 & 2.9 & 96.0 & 161.0 & 118.0 & 103.2 & 49.0 \\
\hline $\mathrm{P} 8^{(120)}$ & 178423 & 3.5 & 96.0 & 158.6 & 116.3 & 106.9 & 51.2 \\
\hline P9 ${ }^{(110)}$ & 110387 & 3.4 & 98.0 & 158.7 & 118.4 & 110.7 & 53.0 \\
\hline
\end{tabular}

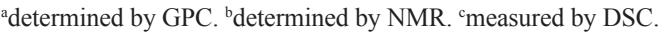


heating ramp with an applied force oscillating at a frequency of $1 \mathrm{~Hz}$. The static force was kept constant at $110 \%$ of the dynamic force. The temperature range analyzed was between $-40^{\circ} \mathrm{C}$ and $230{ }^{\circ} \mathrm{C}$.

MH measurements were conducted on a UHL microhardness tester equipped with a Vickers indenter. Measurements were obtained using an indentation speed of $25 \mu \mathrm{m} / \mathrm{s}$ and a dwell time of $15 \mathrm{~s}$. Samples were analyzed at indentation loads of 10 gf. Ten measurements were recorded for each sample analyzed.

\section{Results and Discussions}

\subsection{Morphological properties}

Table 1 summarizes the polypropylene polymers and fractions used in this study.

\subsubsection{Optical Microscopy analysis}

First, the effect that the molecular weight has on the crystal structure of different polypropylene samples was studied. Figure 1a-c shows OM micrographs of polypropylene fractions $\mathrm{P} 5^{(120)}, \mathrm{P} 4^{(120)}$ and $\mathrm{P} 9^{(110)}$ respectively, which differ in molecular weight (see Table 1). They exhibit a typical $\alpha$-modification spherulite structure of isotactic polypropylenes crystallized from the melt. These micrographs show that all the isotactic polypropylene fractions have well-defined and large $\alpha$-spherulitic morphology. The spherulites grew, impinged on each other, and formed particular polygonal spherulites with clear boundaries. Indications are that, since all the observed spherulites grew at the same rate and their observed size can be considered uniform, the nuclei are formed immediately after cooling to the crystallization temperature and their number remains constant thereafter.

The only effect of the molecular weight that can be noticed in Figure 1 is the slight morphological differences in the sign of birefringence, magnitude of the birefringence and spherulite texture. Second, the effect of tacticity on the crystal structure of different isotactic polypropylenes was studied. Figure 2a-c show OM micrographs of polypropylenes P5, P4 and P14, which differ in their tacticities (Table 1). The graphs show that, under similar crystallization conditions, the dimensions of the crystal structures of these different isotactic polypropylene samples decrease in size with tacticity. This effect can be explained by the restriction of movement of polymer chains caused by chain defects in low tacticity polymers during the crystallization process, resulting in slower crystallization and hence the formation of smaller spherulites. Similar results were obtained in other studies ${ }^{[22-24]}$.

\subsubsection{Scanning Electron Microscopy analysis}

Figure 3a-c shows SEM micrographs of the typical crystallization morphologies of the isotactic polypropylene fractions $\mathrm{P} 5^{(120)}, \mathrm{P} 4^{(120)}$ and $\mathrm{P} 9^{(110)}$ respectively, which differ in molecular weight. All these isotactic polypropylenes
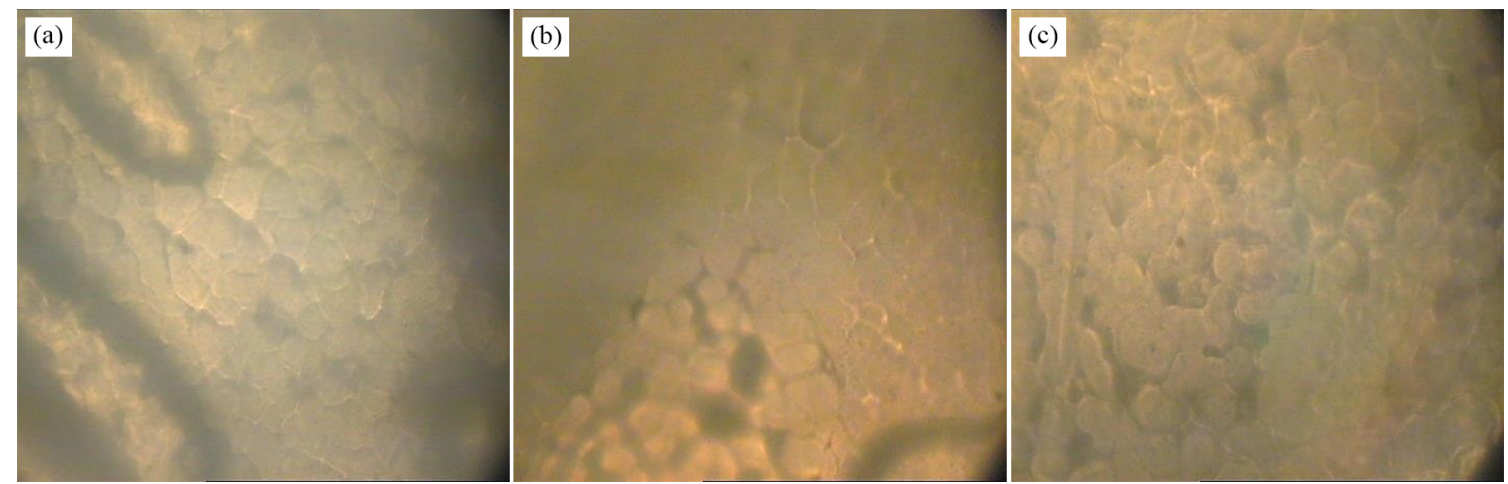

Figure 1. Optical micrographs of isotactic polypropylene fractions: (a) $P 5^{(120)}\left(M_{w}=207823 \mathrm{~g} / \mathrm{mol}\right)$, (b) $P 4^{(120)}\left(M_{w}=195693 \mathrm{~g} / \mathrm{mol}\right)$ and (c) $\mathrm{P}^{(110)}\left(\mathrm{M}_{\mathrm{w}}=110387 \mathrm{~g} / \mathrm{mol}\right)(500 \mathrm{x}$ magnification).
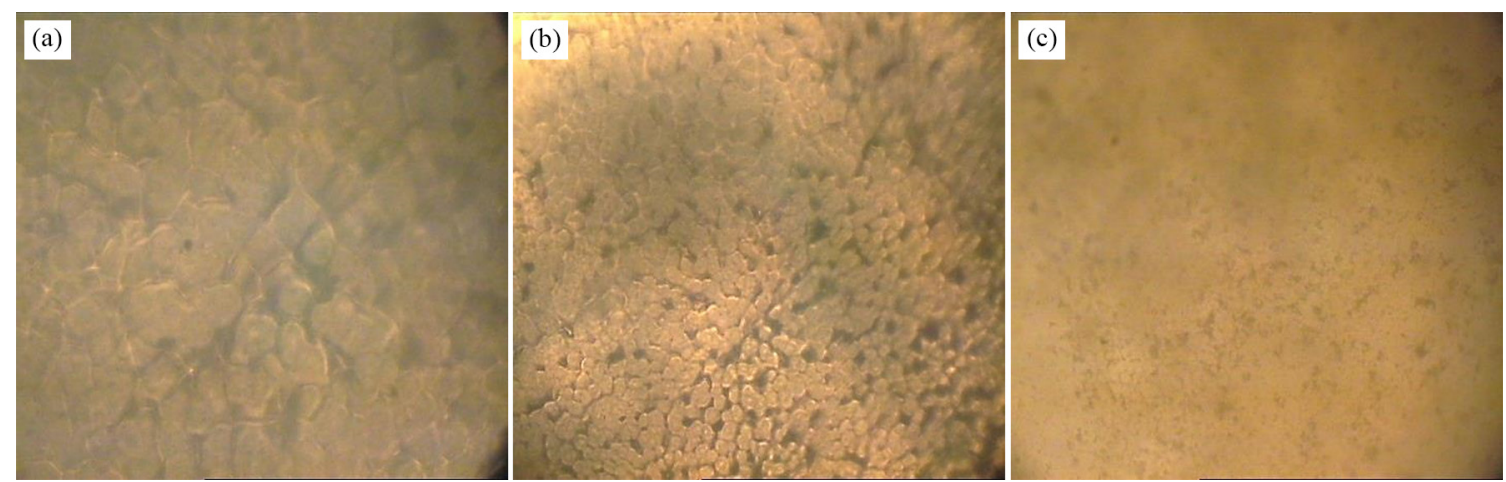

Figure 2. Optical micrographs of isotactic polypropylene polymers: (a) P5 ( $\mathrm{mmmm}=96 \%)$, (b) P4 ( $\mathrm{mmmm}=94 \%)$ and (c) P14 $(\mathrm{mmmm}=86 \%)(500 \mathrm{x}$ magnification $)$. 
revealed well-defined and large spherulitic morphology, comprising a mixture of $\alpha 1$ (disordered) and $\alpha 2$ (ordered) crystal form structures. The spherulites grew, impinged on each other, and formed particular polygonal spherulites with clear boundaries. Moreover, one can clearly see the individual lamellae and lamellar branching structure in the SEM micrographs in Figure 3. The samples consist of crosshatch-type lamellar branching structures, which is the typical characteristic of the $\alpha$ crystal form of isotactic polypropylenes ${ }^{[25,26]}$. In contrast to OM results, clear differences can be distinguished between the three different isotactic polypropylene samples shown in Figure 3. These differences exist in the variety of spherulite sizes and spherulite types classified by their appearance, including the sign and nature of birefringence and crystal lattice. The average diameter of $\mathrm{P}^{(120)}$ spherulites is about 5-15 $\mu \mathrm{m}$ (Figure 3a). Smaller dominant $\alpha$ spherulites (about 5-10 $\mu \mathrm{m}$ ) are observed for $\mathrm{P} 4^{(120)}$ and $\mathrm{P} 9^{(110)}$ (Figure 3b, c respectively).

Figure 4a-c illustrates SEM micrographs of polypropylenes (P5, P4 and P14) that differ in their tacticities (Table 1). Similar to those results obtained from OM above, SEM also shows in Figure 4, that the sizes of the spherulites were decreased drastically with decreasing tacticity. In addition, with decreasing tacticity, the spherulites showed less perfection and the sharp spherulite boundaries became more diffuse (Figure 4c).

The sample P5 with $96.0 \%$ tacticity has the biggest spherulite sizes $(15-25 \mu \mathrm{m})$ while samples P4 and P14 with $94.0 \%$ and $86.0 \%$ tacticities have spherulite sizes about 5-15 and 1-3 $\mu \mathrm{m}$ respectively. SEM micrographs (Figure $4 \mathrm{a}, \mathrm{b}$ ) also show small dimples on the etched surface of samples P5 and P4 may grow to craters and holes. This is due to the extractions of the rubbery materials by the etchant solution $^{[19,20,27,28]}$.

\subsection{Mechanical properties}

In order to correlate the structure of the polypropylene polymers with the mechanical properties, the samples were analyzed using microhardness and DMA.

\subsubsection{Microhardness}

According to literature ${ }^{[29-33]}$, all the parameters that lead to an increase of crystallinity and crystallite sizes (lamellar thickness) will also lead to higher MH values. Hence, the higher the isotacticity, the greater the $\mathrm{MH}$ values obtained. The effect of the molecular weight and molecular weight distribution on the crystallinity of the polypropylene samples $\mathrm{P} 3, \mathrm{P} 4, \mathrm{P} 5, \mathrm{P} 8, \mathrm{P} 17, \mathrm{P} 4^{(120)}$ and $\mathrm{P} 8^{(120)}$ was investigated and results are illustrated in Figure 5. The samples of low molecular weight generally have a broader molecular weight distribution, and vice versa. The molecular weight distribution has an effect on the crystallinity: the samples with a higher degree of crystallinity have a lower molecular weight distribution, and samples with lower degree of crystallinity have a higher molecular weight distribution. On the other hand, there is a slight increase in the crystallinity of the samples with an increase in the molecular weight.

Figure 6 shows the combined effect of the molecular weight and isotacticity on the degree of crystallinity of the polypropylene samples $\mathrm{P} 3, \mathrm{P} 4, \mathrm{P} 5, \mathrm{P} 8, \mathrm{P} 17, \mathrm{P} 4^{(120)}, \mathrm{P} 8^{(120)}$ and $\mathrm{P} 9^{(110)}$. There is a remarkable increase in crystallinity as the isotacticity is increased, from about $93 \%$ to $98 \%$.
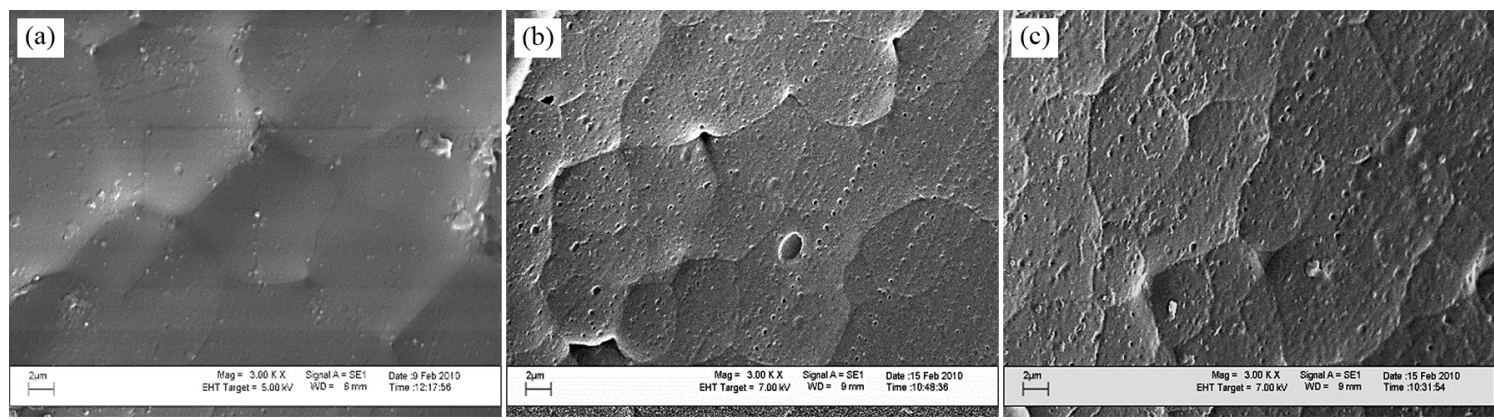

Figure 3. SEM micrographs of isotactic polypropylene fractions: (a) $P 5^{(120)}\left(\mathrm{M}_{\mathrm{w}}=207823 \mathrm{~g} / \mathrm{mol}\right)$, (b) $\mathrm{P} 4^{(120)}\left(\mathrm{M}_{\mathrm{w}}=195693 \mathrm{~g} / \mathrm{mol}\right)$ and (c) $\mathrm{P}^{(110)}\left(\mathrm{M}_{\mathrm{w}}=110387 \mathrm{~g} / \mathrm{mol}\right)(3000 \mathrm{x}$ magnification).
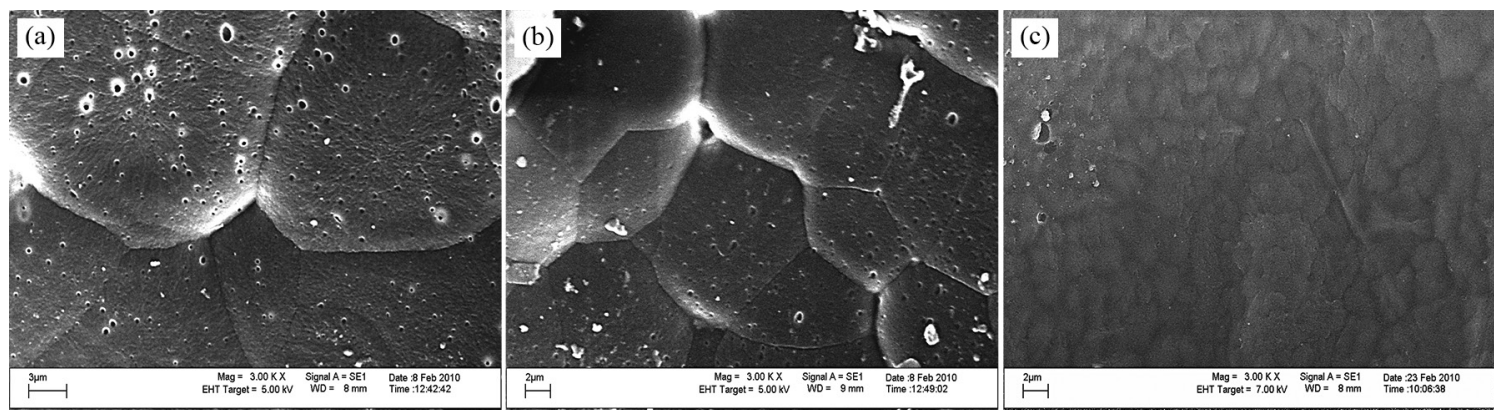

Figure 4. SEM micrographs of isotactic polypropylene polymers: (a) P5 ( $m m m m=96 \%)$, (b) P4 ( $m m m m=94 \%)$ and (c) P14 ( $m m m m=86 \%)$ (3000× magnification). 
In general, the samples with high molecular weight have high isotacticity, as expected, since the more stereospecific sites have a higher propagation constant rate $\left(\mathrm{K}_{\mathrm{p}}\right)$. This is in agreement with the results obtained by Sakurai et al. ${ }^{[34]}$ with regards to the relationship between isotacticity and molecular weight. Moreover, Figures 5 and 6 show that the crystallinity of the samples was largely affected by the isotacticity, which clearly dominates over other effects such as molecular weight and molecular weight distributions. Nevertheless, when the isotacticity values from the polymer samples are similar, the molecular weight and molecular weight distributions exert significant influence on crystallinity.

Looking at the combined effect of the molecular weight and crystallinity on the microhardness of the samples P3, P4, $\mathrm{P} 5, \mathrm{P} 8, \mathrm{P} 17, \mathrm{P} 4^{(120)}, \mathrm{P} 8^{(120)}$ and $\mathrm{P} 9^{(110)}$, as shown in Figure 7 , generally one can see that there is a major increase in the MH with increasing molecular weight and crystallinity of the samples. Since that the most important factor affecting

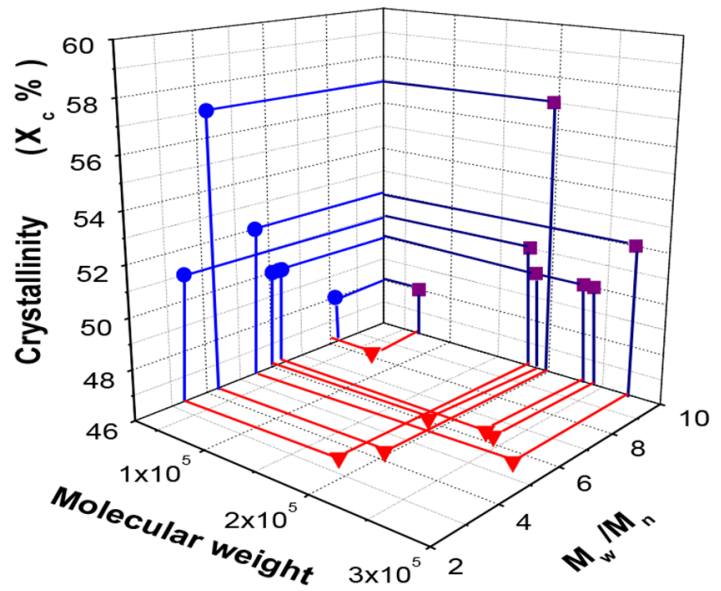

Figure 5. The effect of molecular weight and molecular weight distribution on the crystallinity of the isotactic polypropylene polymers $\mathrm{P} 3, \mathrm{P} 4, \mathrm{P} 5, \mathrm{P} 8, \mathrm{P} 17, \mathrm{P} 4^{(120)}$ and $\mathrm{P} 8^{(120)}$.

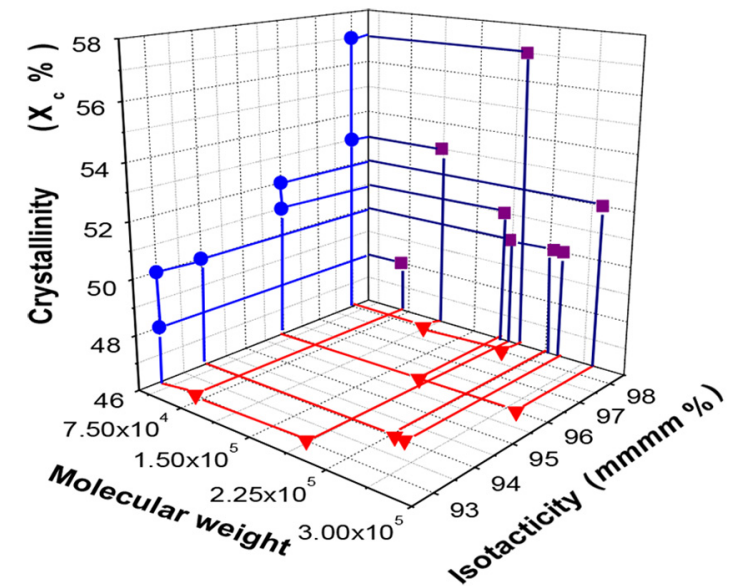

Figure 6. The combined effect of molecular weight and isotacticity on the crystallinity of the isotactic polypropylene polymers P3, P4, $\mathrm{P} 5, \mathrm{P} 8, \mathrm{P} 17, \mathrm{P} 4^{(120)}, \mathrm{P}^{(120)}$ and $\mathrm{P} 9^{(110)}$. the crystallinity of these polypropylene polymers is the isotacticity content, the combined effect of the molecular weight and isotacticity on the $\mathrm{MH}$ of the samples $\mathrm{P} 3, \mathrm{P} 4, \mathrm{P} 5$, $\mathrm{P} 8, \mathrm{P} 17, \mathrm{P} 4^{(120)}, \mathrm{P} 8^{(120)}$ and $\mathrm{P} 9^{(110)}$ is illustrated in Figure 8. It appears that there is a significant increase in the $\mathrm{MH}$ with increasing isotacticities of the samples. Hence, it can be said that the higher the isotacticity the greater the MH. This means that the most important factors affecting the $\mathrm{MH}$ of these polypropylene polymers are those that lead to an increase in crystallinity. In addition, the main parameter which affects the crystallinity can be considered to be the degree of isotacticity of the samples.

It is reasonable that the higher isotacticity content allows easier recrystallization upon the application of an external force to the sample, thus improving the hardness of the sample upon indentation. The magnitude of the effect of the tacticity of the polypropylenes on the properties of the polymer has also been discussed by De Rosa et al. ${ }^{[35]}$.

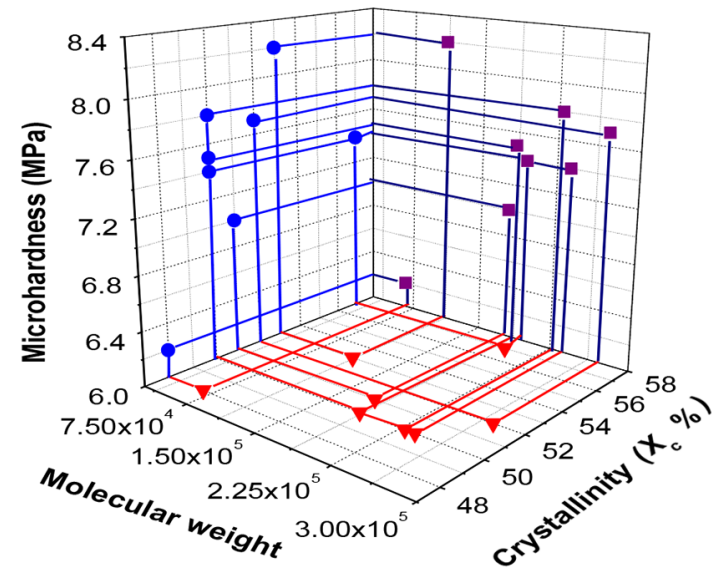

Figure 7. The combined effect of molecular weight and crystallinity on the microhardness of the isotactic polypropylene polymers $\mathrm{P} 3$, $\mathrm{P} 4, \mathrm{P} 5, \mathrm{P} 8, \mathrm{P} 17, \mathrm{P} 4^{(120)}, \mathrm{P}^{(120)}$ and $\mathrm{P} 9^{(110)}$.

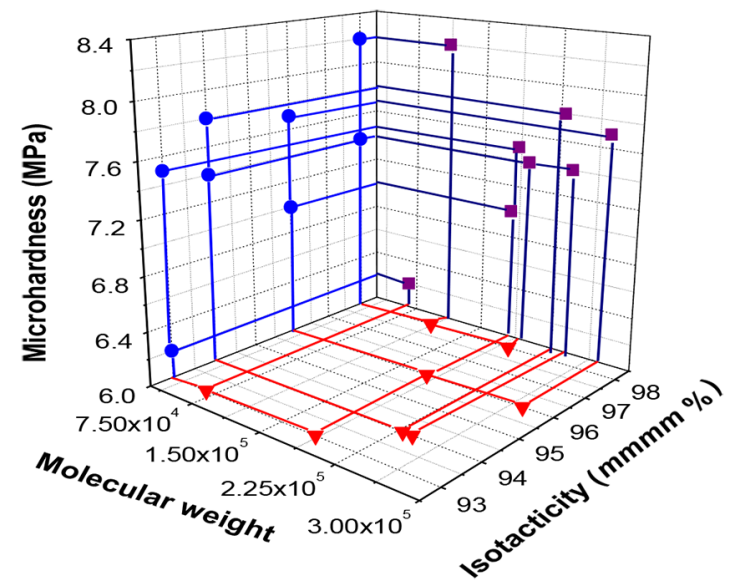

Figure 8. The combined effect of molecular weight and isotacticity on the microhardness of the isotactic polypropylene polymers $\mathrm{P} 3$, P4, P5, P8, P17, P4 ${ }^{(120)}, \mathrm{P}^{(120)}$ and P9 ${ }^{(110)}$. 


\subsubsection{Dynamic Mechanical analysis}

\subsubsection{Effect of molecular weight}

Figure 9 shows the storage modulus of different isotactic polypropylene samples with different molecular weights, as a function in temperature. The storage modulus values increase with increasing molecular weight in the temperature range measured for the different samples. This is in agreement with the results obtained above from the microhardness test. Similar behaviour has been observed by other researchers ${ }^{[8]}$. The reason for the increase in the storage modulus values with increasing molecular weight is due to the higher degree of crystallinity and the presence of a larger number of molecular weight entanglements per chain for the higher molecular weight polymers. Furthermore, an increase in the lamellar thickness as the molecular weight increases also leads to higher storage modulus values ${ }^{[8]}$.

The detailed plot of loss tangent $(\tan \delta)$ of these isotactic polypropylene samples, as a function of the temperature, ranging from -40 to $80^{\circ} \mathrm{C}$ is presented in Figure 10. The tan $\delta$ curves represent the ratio of the ability of the material to store and lose energy, which is sometimes referred to as the clamping ability of a material. It can also be taken as a measurement of the impact properties of the material. It is apparent from Figure 10 that $\beta$-transition, corresponding to the $\mathrm{T}_{\mathrm{g}}$ of isotactic polypropylenes, which occurs over the temperature range $10-20^{\circ} \mathrm{C}$, is slightly shifted to a higher

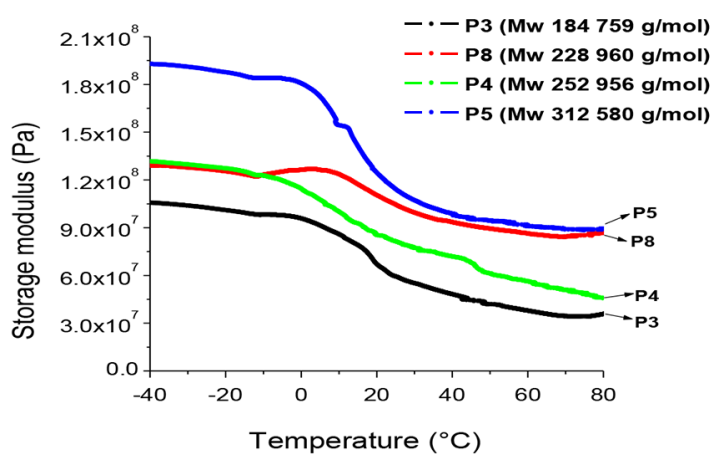

Figure 9. Storage modulus curves as a function of temperature for isotactic polypropylene samples with various molecular weights.

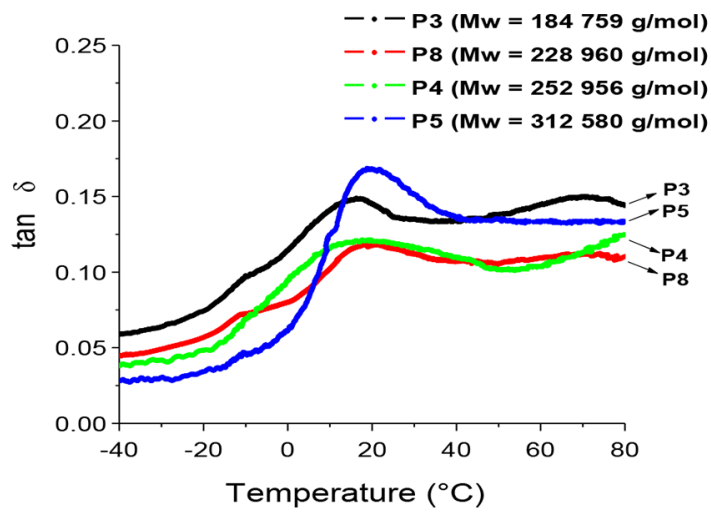

Figure 10. Tan $\delta$ curves as a function of temperature for isotactic polypropylene samples with various molecular weights. temperature as the molecular weight increases $\left(\right.$ from $16^{\circ} \mathrm{C}$ for P3 sample with $\mathrm{M}_{\mathrm{w}} 184759 \mathrm{~g} / \mathrm{mol}$ to $20^{\circ} \mathrm{C}$ for P5 sample with $\mathrm{M}_{\mathrm{w}} 312580 \mathrm{~g} / \mathrm{mol}$ ). The samples with lower molecular weights are less crystalline and therefore contain more amorphous material. The explanation is that the chains have far greater mobility in the amorphous phase in the lower molecular weight samples compared to the samples with higher molecular weights. We do, however, also have to take into consideration the change in the molecular packing in the amorphous phase. Denser packing of the molecular chains leads to a reduction in the molecular motion. The areas of the $\beta$-transitions of the samples, after subtraction of a linear baseline, are given in Figure 11.

Figure 11 shows that the magnitude of the $\beta$-transition increases with increasing molecular weight. Similar results were obtained by Stern et al. ${ }^{[8]}$, who found that the higher molecular weight polymers are generally characterized by larger $\beta$-transition. In fact, a decrease in the mechanical transition of the $\beta$ process is associated with a reduction in the mobility of the polymer chains in the amorphous phase $\mathrm{e}^{[8,36,37]}$.

\subsubsection{Effect of isotacticity}

Figure 12 shows the difference in the storage modulus curves, as a function of temperature, for isotactic polypropylene samples with various isotacticities. Compared to the results obtained from varying the molecular weight, isotactity of isotactic polypropylene samples has further effect on the overall viscoelastic response as shown in Figure 12. The storage modulus is greater in the higher isotactic polypropylene samples than in those with lower tacticity, over the whole temperature range studied $\left(1.51 \times 10^{8} \mathrm{~Pa}\right.$ for $\mathrm{P} 4^{(120)}$ with $98 \mathrm{mmmm} \%$ vs $0.84 \times 10^{8} \mathrm{~Pa}$ for P14 with $86 \mathrm{mmmm} \%$ ) as shown in Figure 12. Moreover, this effect is also observed in the location and intensity of the $\beta$-transition temperature, as shown in Figure 13.

As the isotactic content increases, the location of the $\beta$-transition temperature is considerably shifted to higher temperatures, but its intensity decreases significantly in the higher isotactic polypropylene fractions $\mathrm{P} 5^{(120)}$ and $\mathrm{P} 4^{(120)}$, as can be seen in Figure 14, which shows the areas of the $\beta$-transitions of the different samples. All of these features

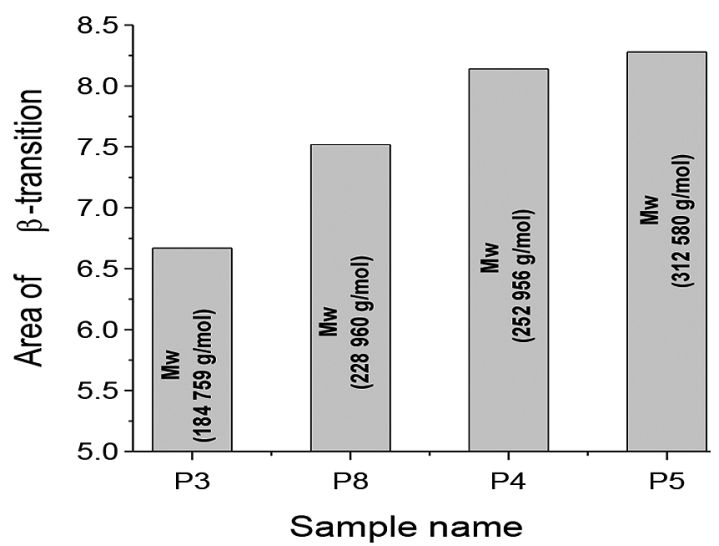

Figure 11. The magnitude of the area of the $\beta$-transition for isotactic polypropylene samples with various molecular weights. 
can be associated with the higher degree of crystallinity that more regular chains can be reached, i.e. as isotacticity is increased in the isotactic polypropylene macromolecules. Therefore, the lowest content of amorphous regions is in the $\mathrm{P} 4^{(120)}$ sample, because its higher crystallinity $\left(\mathrm{X}_{\mathrm{c}}=57 \%\right.$, Table 1) leads to its higher storage modulus, a decrease in magnitude of the $\beta$-transition and the displacement of its location to higher temperatures due to the higher hindrance of motions within the crystalline phase.

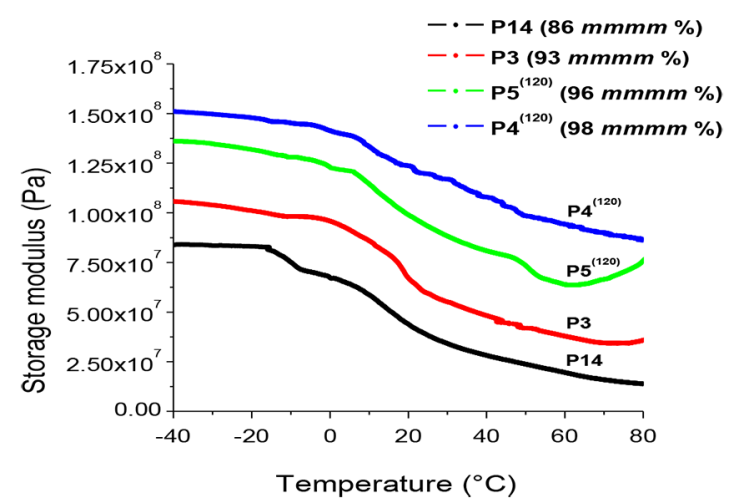

Figure 12. Storage modulus curves as a function of temperature for isotactic polypropylene samples with various isotacticities.

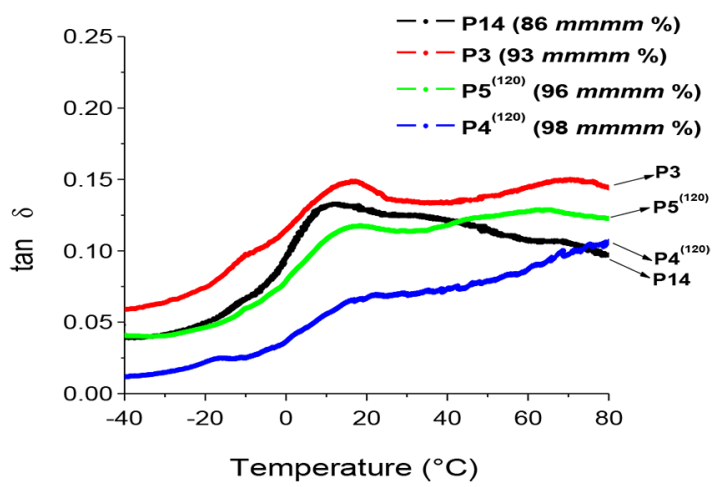

Figure 13. Tan $\delta$ curves as a function of temperature of isotactic polypropylene samples with various isotacticities.

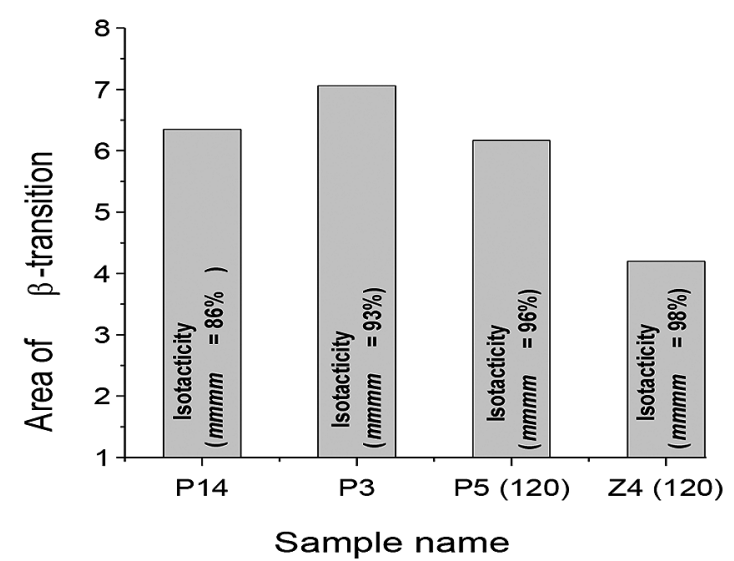

Figure 14. The magnitude of the area of the $\beta$-transition of isotactic polypropylene samples with various isotacticities.

\section{Conclusions}

The relationship between structure and properties was established for different polypropylene samples through their morphological and mechanical characterizations. The effect of isotacticity and molecular weight on the properties of polypropylene samples was investigated. The most important factors affecting the structure and properties of these polypropylene samples are those that lead to an increase of crystallinity. Consequently, the main parameter is the degree of isotacticity, followed by molecular weight. $\mathrm{OM}$ and SEM results showed that all isotactic polypropylene samples had well-defined $\alpha$-spherulitic morphology. OM and SEM also showed that tacticity had a greater effect on the morphological structure of the isotactic polypropylenes than molecular weight. A decrease in isotacticity leads to a clear decrease in the dimensions of the crystal structures for the different isotactic polypropylene samples. Results of the $\mathrm{MH}$ and DMA showed that all the parameters that lead to an increase in crystallinity and crystallite sizes (lamellar thickness) will provide higher MH, storage modulus and $\beta$-transition temperature values. The crystallinity of the samples was shown to be affected by the molecular weight and molecular weight distribution, as well as by the isotacticity of the samples.

\section{Acknowledgements}

The authors would like to thank 1) the International Centre of Macromolecules and Materials Science (Libya) for financial support for Ismael Amer and 2) Mrs Sonja Brandt for the English editing.

\section{References}

1. Moore, J. E. P., \& Larson, G. A. (1996). Introduction to PP business, polypropylene handbook. Munich: Hanser Publishers.

2. Lima, M. F. S., Vasconcellos, M. A., \& Samios, D. (2002). Crystallinity changes in plastically deformed isotactic polypropylene evaluated by $\mathrm{x}$-ray diffraction and differential scanning calorimetry methods. Journal of Polymer Science. Part B, Polymer Physics, 40(9), 896-903. http://dx.doi.org/10.1002/ polb.10159.

3. Asano, T., Calleja, F. J. B., Flores, A., Tanigaki, M., Mina, M. F., Sawatari, C., Itagaki, H., Takahashi, H., \& Hatta, I. (1999). Crystallization of oriented amorphous poly(ethylene terephthalate) as revealed by X-ray diffraction and microhardness. Polymer, 40(23), 6475-6484. http://dx.doi.org/10.1016/S00323861(98)00839-8.

4. Bedia, E., Murakami, S., Kitade, T., \& Kohjiya, S. (2001). Structural development and mechanical properties of polyethylene naphthalate/polyethylene terephthalate blends during uniaxial drawing. Polymer, 42(17), 7299-7305. http:// dx.doi.org/10.1016/S0032-3861(01)00236-1.

5. Hoffman, J. D., \& Miller, R. L. (1988). Test of the reptation concept: crystal growth rate as a function of molecular weight in polyethylene crystallized from the melt. Macromolecules, 21(10), 3038-3051. http://dx.doi.org/10.1021/ma00188a024.

6. Cheng, S. Z., Janimak, J. J., Zhang, A., \& Cheng, H. N. (1990). Regime transitions in fractions of isotactic polypropylene. Macromolecules, 23(1), 298-303. http://dx.doi.org/10.1021/ ma00203a051.

7. Carvalho, B., \& Bretas, R. E. (1998). Quiescent crystallization kinetics and morphology of isotactic polypropylene resins for injection molding. I. Isothermal crystallization. Journal 
of Applied Polymer Science, 68(7), 1159-1176. http://dx.doi org/10.1002/(SICI)1097-4628(19980516)68:7<1159::AIDAPP13>3.0.CO;2-T.

8. Stern, C., Frick, A., \& Weickert, G. (2007). Relationship between the structure and mechanical properties of polypropylene: Effects of the molecular weight and shear-induced structure. Journal of Applied Polymer Science, 103(1), 519-533. http:// dx.doi.org/10.1002/app.24156.

9. Phillips, P. J., \& Vatansever, N. (1987). Regime transitions in fractions of cis-polyisoprene. Macromolecules, 20(9), 21382146. http://dx.doi.org/10.1021/ma00175a016.

10. Cheng, S. Z., Janimak, J. J., Zhang, A., \& Hsieh, E. T. (1991) Isotacticity effect on crystallization and melting in polypropylene fractions: 1. Crystalline structures and thermodynamic property changes. Polymer, 32(4), 648-655. http://dx.doi org/10.1016/0032-3861(91)90477-Z.

11. Janimak, J. J., Cheng, S. Z., Giusti, P. A., \& Hsieh, E. T. (1991). Isotacticity effect on crystallization and melting in polypropylene fractions. II. Linear crystal growth rate and morphology study. Macromolecules, 24(9), 2253-2260. http:// dx.doi.org/10.1021/ma00009a020.

12. Janimak, J. J., Cheng, S. Z., Zhang, A., \& Hsieh, E. T. (1992). Isotacticity effect on crystallization and melting in polypropylene fractions: 3. Overall crystallization and melting behavior. Polymer, 33(4), 728-735. http://dx.doi.org/10.1016/00323861(92)90329-U.

13. De Rosa, C., \& Auriemma, F. (2006). Structural-mechanical phase diagram of isotactic polypropylene. Journal of the American Chemical Society, 128(34), 11024-11025. http:// dx.doi.org/10.1021/ja063464r. PMid:16925410.

14. Lu, H., Qiao, J., Xu, Y., \& Yang, Y. (2002). Effect of isotacticity distribution on the crystallization and melting behavior of polypropylene. Journal of Applied Polymer Science, 85(2), 333-431. http://dx.doi.org/10.1002/app.10637.

15. Bruckner, S., Meille, S. V., Petraccone, V., \& Pirozzi, B. (1991) Polymorphism in isotactic polypropylene. Progress in Polymer Science, 16(2-3), 361-404. http://dx.doi.org/10.1016/00796700(91)90023-E.

16. Samios, D., Tokumoto, S., \& Denardin, E. L. G. (2006) Investigation of the large plastic deformation of iPP induced by plane strain compression: stress-strain behavior and thermomechanical properties. International Journal of Plasticity, 22(10), 1924-1942. http://dx.doi.org/10.1016/j.ijplas.2006.02.009.

17. Amer, I., \& van Reenen, A. (2009). Fractionation and crystallization of isotactic poly(propylenes) prepared with a heterogeneous transition metal catalysts. Macromolecular Symposia, 282(1), 33-40. http://dx.doi.org/10.1002/masy.200950804.

18. Olley, R. H., Hodge, A. M., \& Bassett, D. C. (1979). A permanganic etchant for polyolefines. Journal of Polymer Science. Part B, Polymer Physics, 17(4), 627-643. http:// dx.doi.org/10.1002/pol.1979.180170406.

19. Freedman, A. M., Bassett, D. C., Vaughan, A. S., \& Olley, R. H. (1986). On quantitative permanganic etching. Polymer, 27(8), 1163-1169. http://dx.doi.org/10.1016/0032-3861(86)90003-0.

20. Olley, R. H., \& Bassett, D. C. (1982). An improved permanganic etchant for polyolefines. Polymer, 23(12), 1707-1710. http:// dx.doi.org/10.1016/0032-3861(82)90110-0.

21. Aboulfaraj, M., Ulrich, B., Dahoun, A., \& G'Sell, C. (1993). Spherulitic morphology of isotactic polypropylene investigated by scanning electron microscopy. Polymer, 34(23), 4817-4825. http://dx.doi.org/10.1016/0032-3861(93)90003-S.

22. Lotz, B., Wittmann, J. C., \& Lovinger, A. J. (1996). Structure and morphology of poly(propylenes): a molecular analysis. Polymer, 37(22), 4979-4992. http://dx.doi.org/10.1016/00323861(96)00370-9.

23. Morrow, D. R., \& Newman, B. A. (1968). Crystallization of lowmolecular-weight polypropylene fractions. Journal of Applied Physics, 39(11), 4944-4950. http://dx.doi.org/10.1063/1.1655891.
24. van der Burgt, F. (2002). Crystallization of isotactic polypropylene: the influence of stereo-defects (Masters thesis). Technical University of Eindhoven, Eindhoven.

25. Park, J., Eom, K., Kwon, O., \& Woo, S. (2001). Chemical etching technique for the investigation of melt-crystallized isotactic polypropylene spherulite and lamellar morphology by scanning electron microscopy. Microscopy and Microanalysis, 7(3), 276-286. http://dx.doi.org/10.1017/S1431927601010285. PMid: 12597818.

26. Wang, J., \& Dou, Q. (2008). Crystallization behaviors and optical properties of isotactic polypropylene: comparative study of a trisamide and a rosin-type nucleating agent. Colloid \& Polymer Science, 286(6-7), 699-705. http://dx.doi.org/10.1007/ s00396-007-1821-7.

27. Olley, R. H., \& Bassett, D. C. (1989). On the development of polypropylene spherulites. Polymer, 30(3), 399-409. http:// dx.doi.org/10.1016/0032-3861(89)90004-9.

28. Rybnikáŕ, F. (1985). Selective etching of polyolefines. I. Isotactic polypropylene. Journal of Applied Polymer Science, 30(5), 1949-1961. http://dx.doi.org/10.1002/app.1985.070300513.

29. Seidler, S., \& Koch. T. (2002). Determination of local mechanical properties of $\alpha$ - and $\beta$ - PP by means of microhardness measurements. Journal of Macromolecular Science, Part B: Physics, 41(4-6), 851-861. http://dx.doi.org/10.1081/MB120013069

30. Arranz-Andrés, J., Peña, B., Benavente, R., Pérez, E., \& Cerrada, M. L. (2007). Influence of isotacticity and molecular weight on the properties of metallocenic isotactic polypropylene. European Polymer Journal, 43(6), 2357-2370. http://dx.doi. org/10.1016/j.eurpolymj.2007.03.034.

31. Koch, T., Seidler, S., Halwax, E., \& Bernstorff, S. (2007). Microhardness of quenched and annealed isotactic polypropylene. Journal of Materials Science, 42(14), 5318-5326. http://dx.doi. org/10.1007/s10853-006-0960-4.

32. Flores, A., Aurrekoetxea, J., Gensler, R., Kausch, H. H., \& Calleja, F. J. B. (1998). Microhardness structure correlation of iPP/EPR blends: influence of molecular weight and EPR particle content. Colloid \& Polymer Science, 276(9), 786-793. http://dx.doi.org/10.1007/s003960050311.

33. Harding, G. W., \& van Reenen, A. J. (2011). Polymerisation and structure-property relationships of Ziegler-Natta catalysed isotactic polypropylenes. European Polymer Journal, 47(1), 70-77. http://dx.doi.org/10.1016/j.eurpolymj.2010.10.019.

34. Sakurai, T., Nozue, Y., Kasahara, T., Mizunuma, K., Yamaguchi, N., Tashiro, K., \& Amemiya, Y. (2005). Structural deformation behavior of isotactic polypropylene with different molecular characteristics during hot drawing process. Polymer, 46(20), 8846-8858. http://dx.doi.org/10.1016/j.polymer.2005.01.106.

35. De Rosa, C., Auriemma, F., Di Capua, A., Resconi, L., Guidotti, S., Camuratie, I., Nifant'ev, I., \& Laishevtsev, I. (2004). Structure-property correlations in polypropylene from metallocene catalysts: stereodefective, regioregular isotactic polypropylene. Journal of the American Chemical Society, 126(51), 17040-17049. http://dx.doi.org/10.1021/ja045684f. PMid: 15612743.

36. Jourdan, C., Cavaille, J. Y., \& Perez, J. (1989). Mechanical relaxations in polypropylene: a new experimental and theoretical approach. Journal of Polymer Science. Part B, Polymer Physics, 27(11), 2361-2384. http://dx.doi.org/10.1002/ polb.1989.090271115.

37. Pluta, M., Bartczak, Z., \& Galeski, A. (2000). Changes in the morphology and orientation of bulk spherulitic polypropylene due to plane-strain compression. Polymer, 41(6), 2271-2288. http://dx.doi.org/10.1016/S0032-3861(99)00364-X. 